\title{
From Colorings to Weavings
}

\author{
E. MIRO $^{a, b, *}$, A. GARCIANO ${ }^{b}$ AND A. ZAMBRANO ${ }^{b}$ \\ ${ }^{a}$ Fakultät für Mathematik, Universität Bielefeld, 33615 Bielefeld, Germany \\ ${ }^{b}$ Department of Mathematics, Ateneo de Manila University, Loyola Heights, 1108 Quezon City, Philippines \\ We present a methodology for constructing weavings from 2-colorings of the plane. In particular, we consider \\ tilings $\mathcal{T}$ of the plane by triangles and their corresponding triangle groups $G$. We derive 2-colorings of $\mathcal{T}$ using the \\ index 2 subgroups of $G$.
}

DOI: $10.12693 /$ APhysPolA.126.560

PACS: 02.20.-a, 61.50.Ah, 89.75.Kd

\section{Introduction}

In Ref. [1] Ramsden et al. showed that crystalline nets in the Euclidean space can be constructed from reticulations of the hyperbolic plane $\mathbb{H}^{2}$. The construction of crystalline three-dimensional Euclidean nets is done by projecting two-dimensional hyperbolic tilings onto a family of triply periodic minimal surfaces.

Among the goals of this study is to construct weavings in $\mathbb{H}^{2}$ which can then be used to construct three-periodic patterns. We define a weaving as an interlacement, determined by a weaving map, of two disjoint geometrically identical nets. In the following, we discuss and illustrate the methodology using tilings in the Euclidean plane $\mathbb{E}^{2}$ and, consequently, weavings in $\mathbb{E}^{2}$, but the methodology also works for the hyperbolic plane $\mathbb{H}^{2}$ with no adjustments.

\section{Tilings and their colorings}

Let $\Delta$ be any triangle in $\mathbb{E}^{2}$ with interior angles $\pi / p$, $\pi / q, \pi / r$, where $p, q, r$ are integers $\geq 2$. Repeatedly reflecting $\Delta$ on its sides results in a triangle tiling $\mathcal{T}:=\mathcal{T}(p, q, r)$ of the appropriate plane by copies of $\Delta$. Let $P, Q, R$, respectively, denote the reflections on the sides of $\Delta$ opposite the angles $\pi / p, \pi / q, \pi / r$. The group $G:=G(p, q, r)$ of isometries generated by $P, Q, R$ is called a triangle group and has group presentation $\left\langle P, Q, R \mid P^{2}=Q^{2}=R^{2}=(Q R)^{p}=(R P)^{q}=(P Q)^{r}\right\rangle$.

The group $G$ acts on $\mathbb{E}^{2}$, and the orbit of a point $x \in \mathbb{E}^{2}$ is the set of the images of $x$ under the elements of $G$, denoted by $G x=\{g x \mid g \in G\}$. The set of orbits of $\mathbb{E}^{2}$ under $G$ form a partition of $\mathbb{E}^{2}$. A subset of $\mathbb{E}^{2}$ which contains exactly one point from each of these orbits is called a fundamental region for the action of $G$ on $\mathbb{E}^{2}$ or, in short, a fundamental region of $G$.

It is easy to see that the triangle $\Delta$ contains exactly one point from the orbits of $G$ (except on its boundary which may contain duplicates of representative of some orbits). Then $\Delta$ is a fundamental region of $G$.

The tiling $\mathcal{T}$ is the $G$-orbit of $\Delta$, and $G$ acts transitively on $\mathcal{T}$ and $\operatorname{Stab}_{G} \Delta=\{e\}$. Consequently, there exists a

*corresponding author; e-mail: eprovido@math.uni-bielefeld.de one-to-one correspondence between $G$ and $\mathcal{T}$ given by $g \mapsto g \Delta$ where $g \in G$, and $g^{\prime} \in G$ acts on $g \Delta \in \mathcal{T}$ by sending it to its image under $g^{\prime}$.

Suppose $H$ is a subgroup of $G$ of index $n$. Let $\left\{g_{1}, g_{2}, \ldots, g_{n}\right\}$ be a complete set of left coset representatives of $H$ in $G$ with $g_{1} \in H$, and $\left\{c_{1}, c_{2}, \ldots, c_{n}\right\}$ a set of $n$ colors. Then the assignment $g_{i} H \Delta \mapsto c_{i}$ defines an $n$-coloring of $\mathcal{T}$ which is $G$-transitive.

To construct the coloring, assign color $c_{i}$ to $g_{i} H \Delta$ for $i=1,2, \ldots, n$. The group $G$ acts transitively on $\left\{g_{1} H \Delta, g_{2} H \Delta, \ldots, g_{n} H \Delta\right\}$ with $g \in G$ sending $g_{i} H \Delta$ to $g g_{i} H \Delta$. The elements of $G$ which fix $c_{1}$ constitute the subgroup $H$, while the elements which fix color $c_{i}$ constitute the conjugate subgroup $g_{i} H_{i}^{-1}$ of $H$. Thus, the symmetry group of the coloring is the intersection of all conjugate subgroups of $H$ which is the $\operatorname{Core}_{G} H=$ $\cap g_{i} H g_{i}^{-1}$. This connection between subgroups and coloring is described in [2,3].

A fundamental region of $H$ denoted by $\Delta_{H}$ consists of $n$ copies of $\Delta$ representing each color. Henceforth, we let $H$ be an index two subgroup of $G$. Then a fundamental region $\Delta_{H}$ may be given by $\Delta \cup g \Delta$ for some $g \notin H$; specifically, $\Delta_{H}$ consists of $c_{1}=$ white and $c_{2}=$ black copies of $\Delta$. Furthermore, we impose the following condition for the choice of $g \notin H$ in $\Delta_{H}=\Delta \cup g \Delta$. The reason for imposing this condition will be explained later.

FR Condition. If $H$ is generated by the set $\left\{h_{1}, h_{2}, \ldots, h_{l}\right\}$, we choose $g$ such that the fixed set of each $h_{j},\left\{x \in \mathbb{E}^{2} \mid h_{j} x=x\right\}$, intersect both $\Delta$ and $g \Delta$.

Let us note that for any index 2 subgroup $H$ of $G$, it is possible to choose a generating set of $H$ such that the FR Condition is satisfied. However, this is not necessarily true of higher index subgroups of $G$.

In Fig. 1 (left), we illustrate the tiling $\mathcal{T}:=\mathcal{T}(4,4,2)$ which has fundamental region $\Delta$ with interior angles $\pi / 4$, $\pi / 4, \pi / 2$. It is associated to the triangle group $G:=$ $G(4,4,2)$. Then in Fig. 1 (right), we have the 2-coloring of $\mathcal{T}$ corresponding to the subgroup $H_{2}=\langle R, Q, P R P\rangle$ of $G$. The symmetry group of the coloring Core $_{G} H_{2}$ is $\mathrm{H}_{2}$ itself since $\mathrm{H}_{2}$ is normal in $G$. A fundamental region of $H_{2}$ is $\Delta_{H_{2}}=\Delta \cup P \Delta$, which consists of a white tile $\Delta$ and a black tile $P \Delta$. Let us note that $\Delta_{H_{2}}$ satisfies the FR Condition as the fixed lines of the reflections $R, Q$, $P R P$ intersect both $\Delta$ and $P \Delta$. 


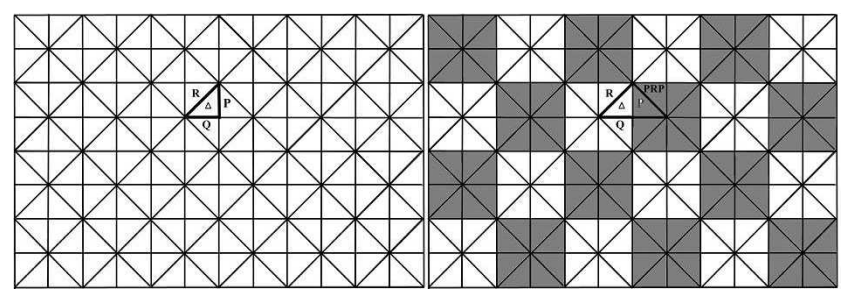

Fig. 1. The tiling $\mathcal{T}(4,4,2)$ (left), and its 2-coloring by $\mathrm{H}_{2}=\langle R, Q, P R P\rangle$ (right).

\section{From colorings to nets}

Given a 2-coloring of the tiling $\mathcal{T}$ associated with a subgroup $H$ of $G$, we simultaneously construct two disjoint nets which are geometrically identical - a black $(B$-)net and a white $(W-)$ net, which we collectively call overlapping nets and denote by $\mathcal{O}_{H}$. The $W$-net is represented by a net of dashed lines for obvious reasons.

\subsection{Vertex sets of overlapping nets}

We define $B$ - and $W$-vertex sets for $\mathcal{O}_{H}$ via $B$ - and $W$-patches defined below using ideas from combinatorial tiling theory [4].

Consider the tiles in $\mathcal{T}$ as chambers, and their colors as the chamber classes. We have black and white chamber classes, which are determined by the subgroup $H$ of $G$ used to color $\mathcal{T}$. Furthermore, for each tile $g \Delta$ in $\mathcal{T}$, label the edges opposite the angles $\pi / q, \pi / r, \pi / p$ by $0,1,2$, respectively. Then record the neighbor-relations of the chambers which are formally described by maps $s_{0}, s_{1}$, and $s_{2}$ from the chamber system onto itself [5]. These maps are defined as follows: the map $s_{i}$ sends a chamber $c$ to the chamber across the edge $i$ of $c$.

In our case, the map $s_{i}$ either fixes $(\mathrm{F})$ the colors or interchanges (I) them. Since the maps $s_{i}$ cannot fix the colors simultaneously, we only have 7 types of patches defined in Table.

Each patch type corresponds to an index 2 subgroup of $G:=G(p, q, r)$. In particular, if all of $p, q, r$ are even, then $G$ has seven index 2 subgroups [3], and each of the seven patch types occurs in a 2 -coloring of $T(p, q, r)$.
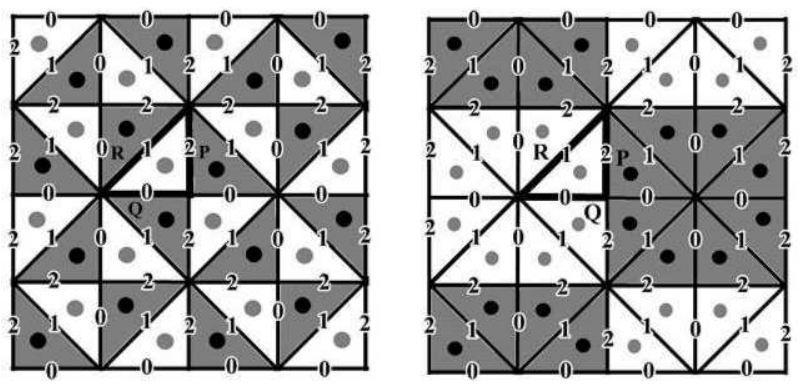

Fig. 2. Patches by: $H_{1}=\langle Q R, R P\rangle$ (left) and $H_{2}=$ $\langle R, Q, P R P\rangle$ (right).

In Fig. 2, we illustrate 2 types of $B$ - and $W$-patches using two of the seven index 2 subgroups of $G(4,4,2)$. The black and grey dots correspond to $B$ - and $W$-vertices, respectively, which are defined below.

Vertex Sets Construction. We define the B-vertex set as the collection of the centroids of the B-patches. The $W$-vertex set is similarly defined.

TABLE

The definition of the 7 types of $B$ - and $W$-patches.

\begin{tabular}{|c|c|c|c|}
\hline$s_{0}$ & $s_{1}$ & $s_{2}$ & Definition of $W$ - and $B$-patches \\
\hline I & $\mathrm{I}$ & $\mathrm{I}$ & $\begin{array}{l}B \text {-patches are the black tiles; } \\
W \text {-patches are the white tiles }\end{array}$ \\
\hline $\mathrm{I}$ & $\mathrm{F}$ & $\mathrm{F}$ & $\begin{array}{l}B \text {-patches are the union of black } \\
\text { tiles about the common vertex } \\
\text { of } 1 \text { - and 2-edges; similarly for } \\
W \text {-patches }\end{array}$ \\
\hline $\mathrm{F}$ & I & $\mathrm{F}$ & $\begin{array}{l}B \text {-patches are the union of black } \\
\text { tiles about the common vertex } \\
\text { of } 0 \text { - and 2-edges; similarly for } \\
W \text {-patches }\end{array}$ \\
\hline F & $\mathrm{F}$ & I & $\begin{array}{l}B \text {-patches are the union of black } \\
\text { tiles about the common vertex } \\
\text { of } 0 \text { - and 1-edges; similarly for } \\
W \text {-patches }\end{array}$ \\
\hline $\mathrm{I}$ & I & $\mathrm{F}$ & $\begin{array}{l}\text { B-patches are the union of ad- } \\
\text { jacent black tiles along 2-edges; } \\
\text { similarly for } W \text {-patches }\end{array}$ \\
\hline I & $\mathrm{F}$ & I & $\begin{array}{l}B \text {-patches are the union of ad- } \\
\text { jacent black tiles along 1-edges; } \\
\text { similarly for } W \text {-patches }\end{array}$ \\
\hline F & $\mathrm{I}$ & $\mathrm{I}$ & $\begin{array}{l}B \text {-patches are the union of ad- } \\
\text { jacent black tiles along } 0 \text {-edges; } \\
\text { similarly for } W \text {-patches }\end{array}$ \\
\hline
\end{tabular}

\subsection{Edges of overlapping nets}

To construct the edges of $\mathcal{O}_{H}$ corresponding to the 2-coloring of the tiling $\mathcal{T}$ by $H$, impose a motif on the fundamental region $\Delta_{H}=\Delta \cup g \Delta$ of $H$, where $g$ is chosen such that the FR Condition is satisfied.

Motif Construction. Suppose $\left\{h_{1}, h_{2}, \ldots, h_{l}\right\}$ is a generating set of $H$. Consider the $B$ - and $W$-vertices on $\Delta_{H}$, and denote them by $b$ and $w$, respectively. For each generator $h_{i}$, connect the vertex $b$ to the vertices $h_{i} b$ and $h_{i}^{-1} b$ using straight line segments. Similarly, connect $w$ to $h_{i} w$ and $h_{i}{ }^{-1} w$. The segment of the edges $\left(b, h_{i} b\right),\left(b, h_{i}^{-1} b\right),\left(w, h_{i} w\right),\left(w, h_{i}^{-1} w\right)$ within $\Delta_{H}$ together with the vertices $w$ and $b$ is the desired motif $\Delta_{m H}$ for $\mathcal{O}_{H}$. The edges $\left(w, h_{i} w\right)$ and $\left(w, h_{i}{ }^{-1} w\right)$ are represented by dashed line segments.

Because of the FR Condition imposed on the choice of the fundamental region $\Delta_{H}$ of $H$, we are assured that the tiles containing the images of vertices $w$ and $b$ intersect $\Delta_{H}$ and the resulting edges are (possibly) minimized. We place the $B$-edges above the $W$-edges whenever they intersect in $\Delta_{m H}$ as in Fig. 3.

We remark that the motif $\Delta_{m H}$ is dependent on $H$ and on the set of generators chosen for $H$. For example, a subgroup $H_{1}$ of $G(4,4,2)$ may be generated by $H_{1 a}=\langle Q R, R P\rangle, H_{1 b}=\langle Q R, P Q\rangle$, or $H_{1 c}=\langle R P, P Q\rangle$. These generating sets result in the following fundamental 

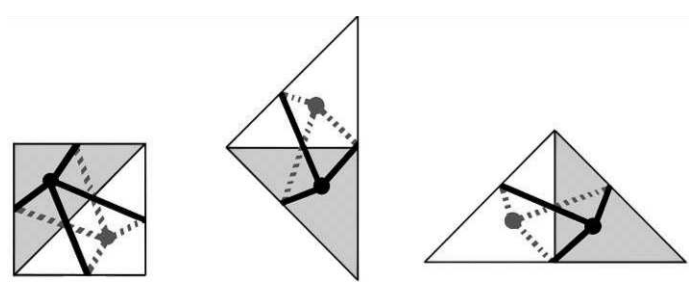

Fig. 3. The motifs derived from three fundamental regions (background) of $H_{1}: \Delta_{m H_{1 a}}$ (left), $\Delta_{m H_{1 b}}$ (middle), $\Delta_{m H_{1 c}}$ (right).

regions satisfying the FR Condition: $\Delta_{H_{1 a}}=\Delta \cup R \Delta$, $\Delta_{H_{1 b}}=\Delta \cup Q \Delta$, and $\Delta_{H_{1 c}}=\Delta \cup P \Delta$, respectively. They yield three distinct motifs shown in Fig. 3.

Overlapping Nets Construction. To get the overlapping nets $\mathcal{O}_{H}$, we let $H$ act on $\Delta_{m H}$. When black edges abut, they are joined continuously to form the $B$-lines of the B-net; similarly, when dashed edges abut, they are joined continuously to form the $W$-lines of the $W$-net.

We denote by $\mathcal{L}_{b}$ and $\mathcal{L}_{w}$ the sets of $B$-lines and $W$-lines (or line segments), respectively, of $\mathcal{O}_{H}$. Now, consider a subset $\mathcal{I}$ of the set $\mathcal{L}_{b} \times \mathcal{L}_{w}$ defined as:

$$
\mathcal{I}=\left\{\left(\ell_{b}, \ell_{w}\right) \in \mathcal{L}_{b} \times \mathcal{L}_{w} \mid \ell_{b} \text { and } \ell_{w}\right.
$$

are intersecting lines\}.

Moreover, we interpret $\left(\ell_{b}, \ell_{w}\right)$ as the intersection point of the lines $\ell_{b}$ and $\ell_{w}$. This means that the set $\mathcal{I}$ is precisely the set of all intersection points of the $B$ - and $W$-lines in $\mathcal{O}_{H}$. We now define a weaving using these notations.

Definition 1. A weaving is a triple $\left(\mathcal{O}_{H}, \mathcal{I}, \omega\right)$ where $\omega$ is a map from $\mathcal{I}$ to the two-point set $\{\oplus, \ominus\}$, called a weaving map.

Let $\left(\ell_{b}, \ell_{w}\right) \in \mathcal{I}$. If $\omega\left(\ell_{b}, \ell_{w}\right)=\oplus$, then we say that $\ell_{b}$ is above $\ell_{w}$; while if $\omega\left(\ell_{b}, \ell_{w}\right)=\ominus$, we say that $\ell_{b}$ is below $\ell_{w}$.

In other words, a weaving is merely an interlacement of the $B$ - and $W$-nets in $\mathcal{O}_{H}$. Denote a weaving derived from the subgroup $H$ with respect to a set of generators of $H$ by $\left(\mathcal{O}_{H}, \mathcal{I}, \omega\right)$ or, simply, $\mathcal{W}_{H}$.

In the next section, we construct the final component of a weaving $\mathcal{W}_{H}$, which is the weaving map $\omega$.

\section{From nets to weavings}

Consider a motif $\Delta_{m H}$ of overlapping nets $\mathcal{O}_{H}$. The intersection points of the $B$ - and $W$-edges within $\Delta_{m H}$ are referred to as IPs, and IPs in the same orbit of $H$ are said to be identifiable. Let us note that the elements of the set $\mathcal{I}$ are just images of the IPs under the elements of $H$. Hence, if we define the weaving map $\omega$ on the IPs, then we can naturally extend the mapping to $\mathcal{I}$.

Weaving Construction. Define $\omega$ on the IPs of $\Delta_{m H}$ by assigning $\oplus$ and $\ominus$ to them. If there are identifiable IPs, assign an identical symbol to each one of them. Then, let $H$ act on the decorated $\Delta_{m H}$, and assign values $(\oplus$ or $\ominus)$ to the rest of the intersection points in $\mathcal{I}$ as fol- lows: if an IP is given a certain symbol, then the elements of its orbit under $H$ will be given the same symbol.

Clearly, the following scenarios will not yield a (proper) weaving.

\section{No Weaving Scenarios (NWS)}

1. $\Delta_{m H}$ has only one IP.

2. All the IPs of $\Delta_{m H}$ are identifiable.

We now outline the methodology for constructing weavings from 2-colorings.

From Colorings to Weavings: The Methodology

1. Consider a tiling $\mathcal{T}:=\mathcal{T}(p, q, r)$ by triangles with interior angles $\pi / p, \pi / q, \pi / r$, together with its corresponding triangle group $G:=G(p, q, r)$.

2. Get a 2-coloring of $\mathcal{T}$ using an index 2 subgroup $H$ of $G$. The symmetry group of such coloring is Core $_{G} H=H$.

3. Choose the fundamental region of $H$ to be $\Delta_{H}=$ $\Delta \cup g \Delta$ where $g \notin H$ and $g$ is chosen such that $F R$ Condition is satisfied.

4. Identify the $B$-and $W$-patches using the chamber system, and then perform Vertex Set Construction.

5. Execute Motif Construction to get the motif $\Delta_{m H}$. Then, construct the overlapping nets $\mathcal{O}_{H}$ via Overlapping Nets Construction.

6. If none of the NWS occurs, carry out Weaving Construction. Otherwise, consider an index 2 subgroup $N$ of $H$.

The motif $\Delta_{m N}$ is composed of two copies of $\Delta_{m H}$, effectively yielding more IPs. The process is repeated if any of the $N W S$ occurs on $\Delta_{m N}$, either by considering another index 2 subgroup of $H$ or an index 2 subgroup of $N$.

\subsection{Equivalent weavings}

We now define when two weavings are equivalent. The following is adapted from the definition given in [6].

Definition 2. Let $H$ and $H^{\prime}$ be subgroups of $G$ which, respectively, yield the weavings $\mathcal{W}_{H}=\left(\mathcal{O}_{H}, \mathcal{I}, \omega\right)$ and $\mathcal{W}_{H^{\prime}}=\left(\mathcal{O}_{H^{\prime}}, \mathcal{I}^{\prime}, \omega^{\prime}\right)$. Let $\mathcal{L}_{b}$ and $\mathcal{L}_{w}$ be the sets of $B$ and $W$-lines in $\mathcal{O}_{H}$, and $\mathcal{L}_{b}^{\prime}$ and $\mathcal{L}_{w}^{\prime}$ be the sets of $B$ and $W$-lines in $\mathcal{O}_{H^{\prime}}$.

We say that $\mathcal{W}_{H}$ and $\mathcal{W}_{H^{\prime}}$ are equivalent weavings if there is a one-to-one correspondence $\phi$ between the elements of $\mathcal{L}_{b}$ and $\mathcal{L}_{b}^{\prime}$, and between the elements of $\mathcal{L}_{w}$ and $\mathcal{L}_{w}^{\prime}$ under which

1. $\mathcal{O}_{H}$ and $\mathcal{O}_{H^{\prime}}$ are combinatorially equivalent, and

2. $\omega\left(\ell_{b}, \ell_{w}\right)=\omega^{\prime}\left(\phi\left(\ell_{b}, \ell_{w}\right)\right) \forall\left(\ell_{b}, \ell_{w}\right)_{H} \in \mathcal{I}$, or $\omega\left(\ell_{b}, \ell_{w}\right)=\overline{\omega^{\prime}\left(\phi\left(\ell_{b}, \ell_{w}\right)\right)} \forall\left(\ell_{b}, \ell_{w}\right)_{H} \in \mathcal{I}$, where $\bar{\oplus}=\ominus$ and $\bar{\ominus}=\oplus$. 
The equivalence classes of weavings with respect to this equivalence relation are called weaving patterns.

It is straightforward to show that if a weaving $\mathcal{W}$ can be obtained from a weaving $\mathcal{W}^{\prime}$ by some isometry of the plane, then they are equivalent weavings. For example, consider the weavings generated by the subgroup $H_{1}$ using its two sets of generators $H_{1 b}=\langle Q R, P Q\rangle$ and $H_{1 c}=\langle R P, P Q\rangle$ shown in Fig. 4. Observe that if we rotate $\mathcal{W}_{H_{1 b}}$ about the centroid of $\boxplus$ by $90^{\circ}$ clockwise and then reflect along the vertical line passing through that point, we get $\mathcal{W}_{H_{1 c}}$. Thus, $\mathcal{W}_{H_{1 b}}$ and $\mathcal{W}_{H_{1 c}}$ are equivalent.

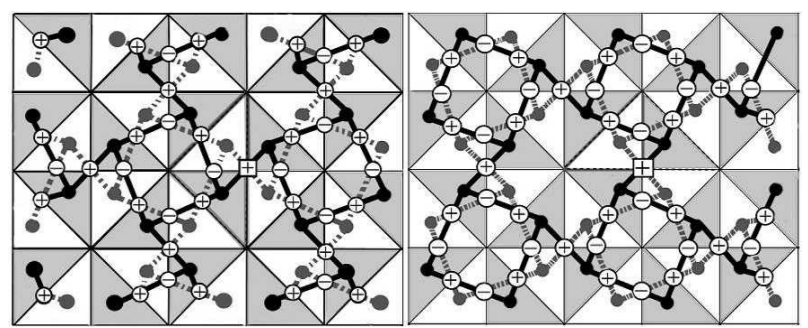

Fig. 4. Equivalent weavings generated by $H_{1}: \mathcal{W}_{H_{1 b}}$ (left), $\mathcal{W}_{H_{1 c}}$ (right).

Suppose that $N$ and $K$ are conjugate subgroups in $G$; that is, $K=g N g^{-1}$ for some $g \in G$. It can be shown that if $\Delta_{N}$ is a fundamental region of $N$, then $g \Delta_{N}$ is a fundamental region of $K$. Consequently, given a weaving $\mathcal{W}_{N}$ from $N$, we can derive a weaving $\mathcal{W}_{K}$ of $K$ which is equivalent to $\mathcal{W}_{N}$. In particular, applying the isometry $g$ on $\mathcal{W}_{N}$ results in the weaving $\mathcal{W}_{K}$

Now suppose a subgroup $H$ of $G$, with fundamental region $\Delta_{H}$, yields a weaving $\mathcal{W}_{H}$ generated by the decorated motif $\Delta_{m H}$. A fundamental region of an index 2 subgroup $N$ of $H$ is a union of two copies of $\Delta_{H}$. Then one can get a weaving $\mathcal{W}_{N}$ by using the union of two copies of the decorated $\Delta_{m H}$. In fact, the weavings $\mathcal{W}_{H}$ and $\mathcal{W}_{N}$ are identical.

We summarize the discussion above in the following proposition.

Proposition 1. Let $H, K, N$ be subgroups of the triangle group.

1. If $\mathcal{W}$ can be obtained from the weaving $\mathcal{W}^{\prime}$ by some isometry of the plane, then they are equivalent weavings.

2. Suppose $K$ and $N$ are conjugate subgroups and $\mathcal{W}_{N}$ is a weaving generated by $N$, then $K$ generates $a$ weaving $\mathcal{W}_{K}$ which is equivalent to $\mathcal{W}_{N}$.

3. Suppose $H$ yields a weaving $\mathcal{W}_{H}$ and $N$ is a subgroup of $H$ of even index, then $N$ yields a weaving $\mathcal{W}_{N}$ which is equivalent (identical) to $\mathcal{W}_{H}$.

\subsection{Example}

The following are some of the weaving patterns generated from $H_{2}=\langle R, Q, P R P\rangle$ and its subgroups.

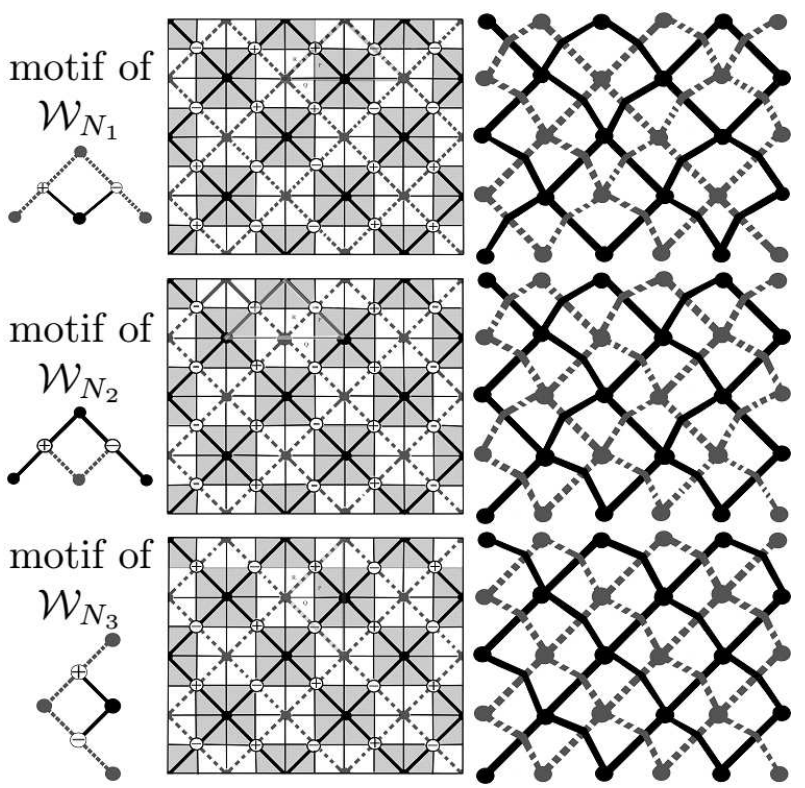

The motif $\Delta_{m H_{2}}$ has only one IP, so we consider the index 2 subgroups of $H_{2}$, and subsequently their index 2 subgroups when NWS occur. We obtain the above non-equivalent weaving patterns from index 4 subgroups of $\mathrm{H}_{2}: \quad N_{1}=\langle Q, R, P R P Q R Q P R P\rangle$, $N_{2}=\langle Q, R P R P, R Q R P R Q R P\rangle$, and $N_{3}=\langle R, Q R Q$, $P R Q R Q P\rangle$. They are all index 2 subgroups of some index 2 subgroups of $\mathrm{H}_{2}$.

\section{Acknowledgments}

The authors would like to thank Stephen Hyde for the helpful suggestions and comments. E. Provido would like to thank Ateneo de Manila University (LS SWF Grant), the Alexander von Humboldt Foundation, and the Collaborative Research Centre 701 at the University of Bielefeld for the generous support.

\section{References}

[1] S.J. Ramsden, V. Robins, S.T. Hyde, Acta Crystallogr. A 65, 81 (2009).

[2] E.D.B Provido, M.L.A.N. De Las Peñas, R. Felix, Acta Crystallogr. A 69, 445 (2013).

[3] M.L.A.N. De Las Peñas, R. Felix, E.D.B. Provido, Z. Kristallogr. 222, 443 (2007).

[4] D.H. Huson, Geometr. Dedic. 47, 269 (1993).

[5] S.J. Ramsden, V. Robins, S.T. Hyde, S. Hungerford, EPINET: Euclidean Patterns in Non-Euclidean Tilings, The Australian National University, Canberra 2005-2009.

[6] J. Pach, R. Pollack, E. Welzl, Algorithmica 9, 561 (1993). 